\title{
Hemothorax induced by cardiac perforation resulting from two active screw-in leads: A case report ${ }^{*}$
}

\author{
Hideki Morita $^{1 \#}$, Tsutomu Saito ${ }^{2}$, Yoshio Misawa ${ }^{2}$ \\ ${ }^{1}$ Department of Cardiovascular Surgery, Saitama Red Cross Hospital, Saitama, Japan \\ ${ }^{2}$ Division of Cardiovascular Surgery, Jichi Medical University, Shimotsuke, Japan \\ Email: ${ }^{\text {uddon@jichi.ac.jp }}$
}

Received 17 January 2013; revised 12 March 2013; accepted 17 April 2013

Copyright (c) 2013 Hideki Morita et al. This is an open access article distributed under the Creative Commons Attribution License, which permits unrestricted use, distribution, and reproduction in any medium, provided the original work is properly cited.

\begin{abstract}
We describe the case of a 77-year-old female who underwent dual-chamber permanent pacemaker implantation using two active screw-in leads for complete atrioventricular block. The two active screw-in leads perforated the atrial and ventricular walls causing cardiac tamponade and a left hemothorax associated with ventricular perforation. An emergency sternotomy was performed to facilitate drainage and hemostasis. Although hemothorax due to pacing lead perforation is very rare, such a possibility should always be considered.
\end{abstract}

Keywords: Pacemaker; Pacing Complications;

Hemothorax; Screw-In Lead

\section{INTRODUCTION}

In 1980, an endocardial atrial screw-in lead was introduced to the market. Since then, a few complications related to its use have been reported, including lead dislodgment, lead fracture, acute pericarditis, and cardiac perforation [1]. Here we present a case of cardiac perforation induced by two active screw-in atrial and ventricular leads that resulted in hemothorax due to an injury caused by the tip of the ventricular lead.

\section{CASE}

A 77-year-old female was implanted with a dual-chamber permanent pacemaker (DDD) for an atrioventricular block. The right atrial and ventricular active screw-in leads were inserted via the left subclavian vein and the device was implanted in the left subclavian area. Ten hours later, pacing failure and dyspnea were observed.

\footnotetext{
*Conflict of interest: none declared.

${ }^{\#}$ Corresponding author.
}

Computed tomography revealed pericardial and left thoracic effusion, with the tip of the ventricular screw-in lead just outside the pericardium and pleura (Figure 1). A diagnosis of cardiac tamponade due to cardiac perforation induced by the pacemaker leads was confirmed and the patient was subsequently referred to our hospital where an emergency sternotomy was performed to achieve drainage and hemostasis. During the surgery, the atrial pacing lead was found perforating the right atrial tissue. When the heart was lifted, the ventricular pacing lead was found perforating the right ventricular tissue and reaching the thoracic wall (Figure 2). Moreover, a left intercostal vein was injured by the lead, inducing a left hemothorax. The tips of both the leads were cut and the remnants pushed into the heart. Thereafter, the remnant leads were extruded out of a subclavian pocket with the permanent pacemaker. Hemostasis was achieved with pledgeted sutures using 4-0 polypropylene covered with a fibrin sheath. An epicardial pacing electrode was placed on the right ventricular wall. Ten days later, a new permanent pacemaker (VVI) was reimplanted in the left

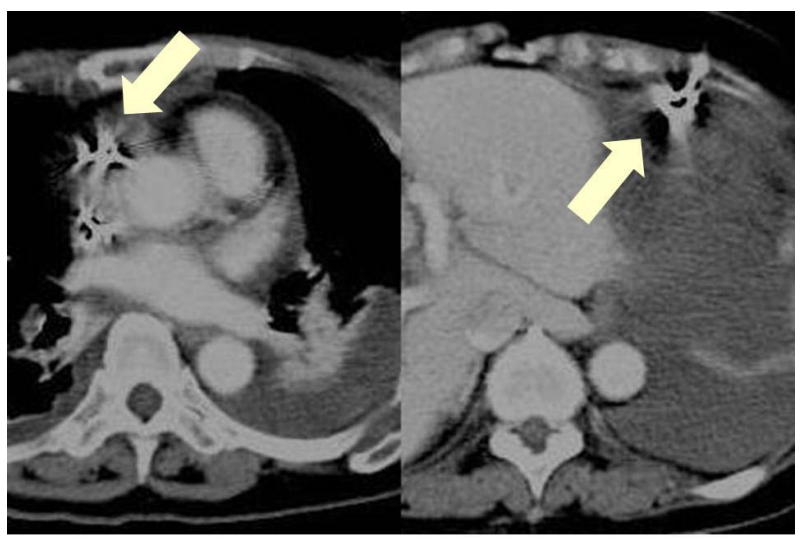

Figure 1. Contrast computed tomography. Left pleural effusion and perforation of the right atrial wall (left); The tip of the ventricular lead is stuck in the left thoracic wall (right). 


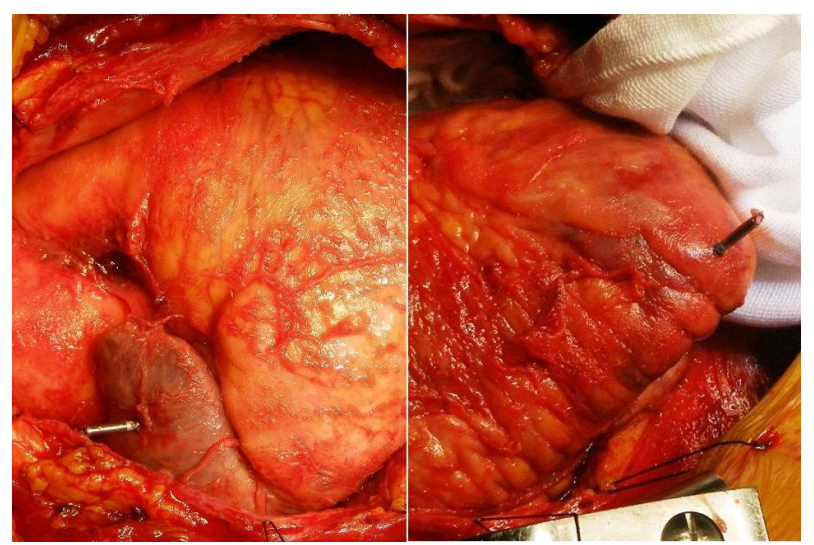

Figure 2. Surgical findings. Two leads can be seen perforating the right atrium and the ventricle.

prepectoral area. The patient was discharged 20 days after surgery and has been asymptomatic since then.

\section{DISCUSSION}

Complications reported in up to $9 \%$ of atrial lead placements are most often related to obtaining venous access; e.g., hemorrhage and pneumothorax (2\%), lead dislodgement (4.2\%), inadequate pacing and sensing (3.5\%), and acute pericarditis (5\% in patients receiving active-fixation atrial leads) [2,3]. Myocardial perforation resulting in pericardial effusion or tamponade is rare and requires management by open heart surgery. Ventricular perforation may seal spontaneously but atrial perforation usually requires open drainage, as closed pericardiocentesis is rarely successful $(<25 \%)[4]$.

The management of myocardial perforations is controversial [5]. In some reported cases, patients were asymptomatic and their pacemaker continued to work despite pericardial perforation; thus, they did not require surgery. Perforation of the thin atrial free wall by the tip of the helix of an active-fixation atrial lead may result in pericardial irritation (pericarditis) or life-threating cardiac tamponade. Thus, the conventional initial management of cardiac tamponade caused by lead perforation is emergency percutaneous pericardiocentesis with placement of a drainage catheter. Once the patient is stabilized, the offending lead is usually repositioned in the operating room where direct visualization and repair of the perforation is possible [6]. In the present case, the patient suffered from dyspnea due to cardiac tamponade and pacing failure; therefore, emergency surgery was performed.

Hemothorax is commonly associated with puncture of the subclavian vein. Few reports document the pacemaker leads perforating both pericardium and pleura, resulting in pneumothorax [2]. However, these were rare cases in which the hemothorax was induced by the endocardial ventricular lead; the tip of the lead injured the thoracic wall through the pericardium and pleura. Hemostasis was achieved directly by performing median sternotomy.

In conclusion, atrial lead perforations and hemothorax due to perforation by a ventricular lead are very rare but represent potentially catastrophic complications. Great caution must be taken when implanting screw-in leads, particularly into the anatomically thin right atrial free wall. When perforation by a lead occurs, extraction of the lead is not mandatory; however, if required, the procedure should preferably be performed using open heart surgery.

\section{REFERENCES}

[1] Greene, T.O., Portnow, A.S. and Huang, S.K.S. (1994) Acute pericarditis resulting from an endocardial active fixation screw-in atrial lead. Pacing and Clinical Electrophysiology, 17, 21-25. doi:10.1111/j.1540-8159.1994.tb01346.x

[2] Dilling-Boer, D., Ector, H., Willems, R. and Heidbuchel, H. (2003) Pericardial effusion and right-sided pneumothorax resulting from an atrial active-fixation lead. $E$ uropace, 5, 419-423. doi:10.1016/S1099-5129(03)00079-5

[3] Sivakumaran, S., Irwin, M.E. and Gulamhusein, S.S. (2002) Postpacemaker implant pericarditis: Incidence and outcomes with active-fixation leads. Pacing and Clinical Electrophysiology, 25, 833. doi:10.1046/j.1460-9592.2002.t01-1-00833.x

[4] Velavan, P. and Chauhan, A. (2003) An unusual presentation of delayed cardiac perforation caused by atrial screw-in lead. Heart, 89, 364. doi:10.1136/heart.89.4.364

[5] Matsushita, A., Komiya, T., Tamura, N. and Sakaguchi, G. (2008) Pneumopericardium caused by a permanent endocardial pacing lead. Interactive Cardiovascular and Thoracic Surgery, 7, 1127-1128. doi:10.1510/icvts.2008.183947

[6] Geyfman, V., Storm, R.H., Lico, S.C. and Oren, J.W. (2007) Cardiac tamponade as complication of active-fixation atrial lead perforations: Proposed mechanism and management algorithm. Pacing and Clinical Electrophysiology, 30, 498-501. doi:10.1111/j.1540-8159.2007.00699.x 